\title{
Collective motion of macroscopic spheres floating on capillary ripples: Dynamic heterogeneity and dynamic criticality
}

\author{
Ceyda Sanl $1,{ }^{1,2, *}$ Kuniyasu Saitoh, ${ }^{3, \dagger}$ Stefan Luding, $, 3, \ddagger$ and Devaraj van der Meer ${ }^{2, \S}$ \\ ${ }^{1}$ CompleXity and Networks, naXys, University of Namur, 5000 Namur, Belgium \\ ${ }^{2}$ Physics of Fluids, University of Twente, P.O. Box 217, 7500 AE Enschede, The Netherlands \\ ${ }^{3}$ Multi Scale Mechanics, University of Twente, P.O. Box 217, 7500 AE Enschede, The Netherlands \\ (Received 15 September 2013; revised manuscript received 7 May 2014; published 30 September 2014)
}

\begin{abstract}
When a densely packed monolayer of macroscopic spheres floats on chaotic capillary Faraday waves, a coexistence of large scale convective motion and caging dynamics typical for glassy systems is observed. We subtract the convective mean flow using a coarse graining (homogenization) method and reveal subdiffusion for the caging time scales followed by a diffusive regime at later times. We apply the methods developed to study dynamic heterogeneity and show that the typical time and length scales of the fluctuations due to rearrangements of observed particle groups significantly increase when the system approaches its largest experimentally accessible packing concentration. To connect the system to the dynamic criticality literature, we fit power laws to our results. The resultant critical exponents are consistent with those found in densely packed suspensions of colloids.
\end{abstract}

DOI: 10.1103/PhysRevE.90.033018

PACS number(s): 47.57.Gc, 64.60.Ht, 68.03.Cd, 83.80.Fg

\section{INTRODUCTION}

Small-scale events can dominate statistical systems to such an extent that one observes phenomena on a global scale. From the classical to the quantum limit, microscopic fluctuations may even change the phase of matter when appropriate control parameters are tuned to critical values [1,2]. Even if their origin and nature are not always understood, these spatiotemporal microscopic fluctuations can drive common observable behavior near to such a phase transition. For classical particulate systems, a vast range of materials exhibits a sudden change to a rigid state called a glass or jamming transition. Thermal systems [3-8], e.g., supercooled liquids near a transition temperature, and emulsions and colloidal suspensions at a critical packing fraction [5,6,8], exhibit a glass transition. Furthermore, athermal systems such as foams and granulates experience a jamming transition, also at a critical packing fraction [9-13]. In all these systems, transient spatial fluctuations lead to a large scale cooperative motion of their constituents near the transition [3-8,14-21].

In this paper we investigate the dynamics of the collective events near the critical density in an alternative experiment: Macroscopic spheres floating on the surface of capillary Faraday waves. Our control parameter is the floating sphere concentration $\phi$ on the surface which is varied from a moderate value, i.e., $\phi=0.65$ to the maximum value attainable experimentally, i.e., $\phi_{\text {expt }}=0.77$. Erratic forces due to the surface waves [22] and the attractive capillary interaction among the spheres [23-25] together make our system markedly different from the previously studied ones [16,17,19,26-28]: A distinct feature is a large scale convection of the spheres on the wave which (for all $\phi$ ) forms naturally and strongly affects the visible dynamics.

\footnotetext{
*ceyda.sanli@unamur.be; cedaysan@gmail.com

${ }^{\dagger}$ k.saitoh@utwente.nl

${ }^{\ddagger}$ s.luding@utwente.nl

§d.vandermeer@utwente.nl
}

We aim to understand to what extent concepts from the glass and jamming literature-such as dynamic heterogeneity and dynamic criticality-still hold in this convective system. To do so we subtract the convective mean flow using a coarse graining (or homogenization) method and analyze the features of our system both before and after this procedure. Recently filtering long time convection in displacement by a stroboscopic technique has been described in the experimental granular literature [28]. The current work however reports a full study, comparing results with and without filtering.

Dynamic heterogeneity investigates the relation between the local dynamic events on the particle scale and the resultant large-scale cooperative motion [3,4,6,7,28-33]. For its quantification two observables are introduced: The four-point dynamic susceptibility $\chi_{4}(\tau)$, a measure of to what extent the single particle dynamics of the system is heterogeneous in space, i.e., fast and slow flow regions present simultaneously, and irregular in time, and the four-point correlation function $g_{4}(r, \tau)$, a measure of how often $(\tau)$ and from how far $(r)$ two arbitrarily chosen single particle dynamics correlate to one another in time and two points in space. The idea behind this is first to capture and characterize locally heterogeneous events and second to determine both for how long and over what distance the local heterogeneity cooperatively dominates the flow. Both quantities $\chi_{4}(\tau)$ and $g_{4}(r, \tau)$ were calculated for colloids [5,14,15], driven hard granulates [16-18,27,3336], and foams [21]. For all cases, the time and length scales and the number of collective events, such as escaping from cages [33] and rearrangements of particle groups [14], dramatically increase near the transition. In spite of the observed quantitative similarities, the many different origins of dynamic heterogeneity summarized in Ref. [28] suggest that we are far from a complete understanding.

The common nature of the behavior of classical particulate systems near transitions encourages to ask whether there is universality. This has led to the concept of dynamic criticality [1719,37-40], which postulates a power-law relation between the (diverging) length and time scales close to the phase transition. The uniqueness of this-and other-exponents in different systems would then support the existence of 

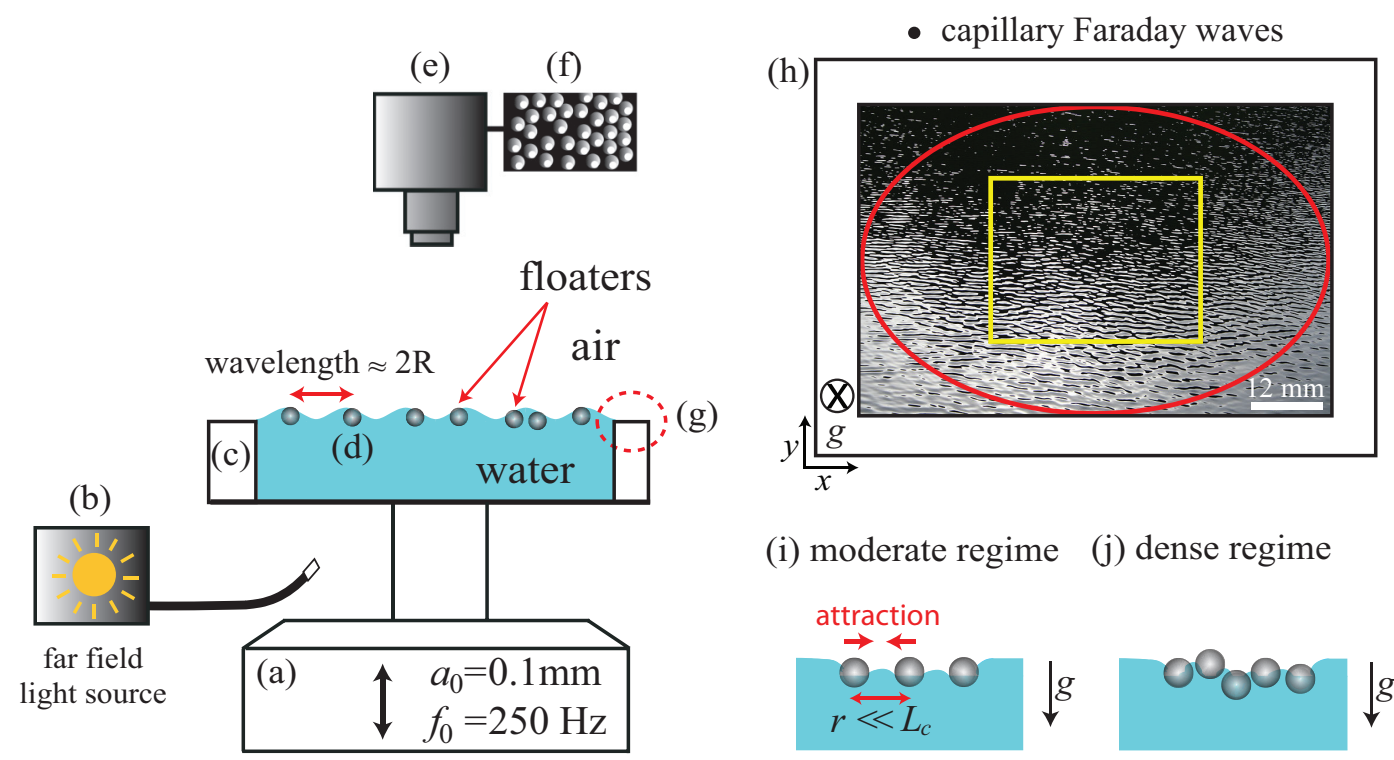

(i) moderate regime (j) dense regime

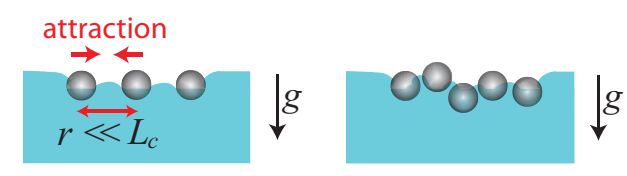

FIG. 1. (Color online) Left panel: Experimental setup: (a) Shaker, (b) far field light source, (c) transparent hydrophilic glass container, (d) hydrophilic spheres, (e) high speed camera, and (f) sample from a camera image for densely packed floater configuration. The advantage of using a far field light source is that all particles can be detected using the white spots in the images. (g) Pinned brim-full boundary condition. Right panel: (h) Top view of capillary Faraday waves, with the elliptic boundary containing the particles (red ellipse) and the field of view of the camera (yellow rectangle). (i) and (j) Sketch of the typical meniscus around the hydrophilic floaters in moderate (i) and expected that in extremely densely packed $(\mathrm{j})$ regimes.

universality [17-19,37-40]. There is evidence pointing to universality in the above sense in various systems [17-19,27]. However, investigation is ongoing [32,41-43]. Furthermore, increasing the number of systems either obeying or disobeying the universality and determining the universality class of the transition are key to reach a more complete understanding.

We will therefore analyze our system in the light of both dynamic heterogeneity and dynamic criticality.

\section{EXPERIMENT}

A schematic illustration of the experimental setup, which is the same one having been already used in our earlier study [25], is shown in Fig. 1. A rectangular container [Fig. 1(a)] is attached to a shaker providing a vertical sinusoidal oscillation such that the vertical position of the container varies as a function of time $t$ as $a_{0} \sin \left(2 \pi f_{0} t\right)$, where $a_{0}$ is the shaking amplitude and $f_{0}$ is the shaking frequency. Here both $a_{0}$ and $f_{0}$ are fixed to $0.1 \mathrm{~mm}$ and $250 \mathrm{~Hz}$, respectively. This combination is chosen to create capillary ripples on the water surface with a wavelength in the order of the floater diameter $(\approx 0.62 \mathrm{~mm})$. The container is filled with purified water (Millipore water with a resistivity $>18 \mathrm{M} \Omega \mathrm{cm}$ ) such that the water level is perfectly matched with the container edge as shown in Fig. $1(\mathrm{~g})$ to create the brim-full boundary condition [44]. Spherical hydrophilic polystyrene floaters [Fig. 1(d)], contact angle $74^{\circ}$, and density $1050 \mathrm{~kg} / \mathrm{m}^{3}$, with an average radius $R$ of $0.31 \mathrm{~mm}$ are carefully distributed over the water surface to make a monolayer of floaters [45]. The polydispersity of the floaters is approximately $14 \%$ and assumed to be just wide enough to avoid crystallization [46]. To avoid any surfactant effects, both the container and the floaters are cleaned by performing the cleaning protocol as described in Ref. [47].
A continuous white fiber light source (Schott) is used to illuminate the floaters from far away as shown in Fig. 1(b). The positions of the floaters are recorded with a high-speed camera (Photron Fastcam SA.1) at 60-500 frames per second. The lens (Carl Zeiss $60 \mathrm{~mm}$ ) is adjusted such that it focuses on the floaters at the nondeformed water surface. Here we use the random capillary Faraday waves to just agitate the densely packed floaters so that there is no macroscopic apparent amplitude observed. The wave amplitude is always considerably smaller than the floater radius $(\approx 0.31 \mathrm{~mm})$.

The resultant capillary ripples on the water surface in the container, made from transparent hydrophilic glass with $10 \mathrm{~mm}$ height and a $81 \times 45 \mathrm{~mm}^{2}$ rectangular cross section, are shown in Fig. 1(h). To eliminate the boundary effects due to the sharp corners of the container, an elliptic rim made from plastic is used to contain the particles. Each image taken with the high speed camera is $512 \times 640 \mathrm{px}^{2}\left(36 \times 28 \mathrm{~mm}^{2}\right)$, where px means pixel, as shown by the yellow rectangle (size ratios are preserved). The horizontal field of view is $\sim 35 \%$ of the total area of the ellipse. Due to the asymmetric surface deformation around each hydrophilic heavy sphere, there is an attraction $[23,24]$ between the spheres so that the floaters are cohesive [48]. For moderate $\phi$, the monolayer can be considered two dimensional [Fig. 1(i)]. In the densely packed regime however [Fig. 1(j)], particles are so close that the layer may (locally) buckle and have three-dimensional aspects [49].

The control parameter of the experiment is the floater (packing) concentration $\phi$, which (ignoring buckling) is measured by determining the area fraction covered by the floaters in the area of interest [Fig. 1(h)]. In this study, $\phi$ is increased from moderate to large concentrations, $\phi=0.65-0.77$.

Under the influence of the erratic capillary waves and the attractive capillary interaction, a large scale convective motion 

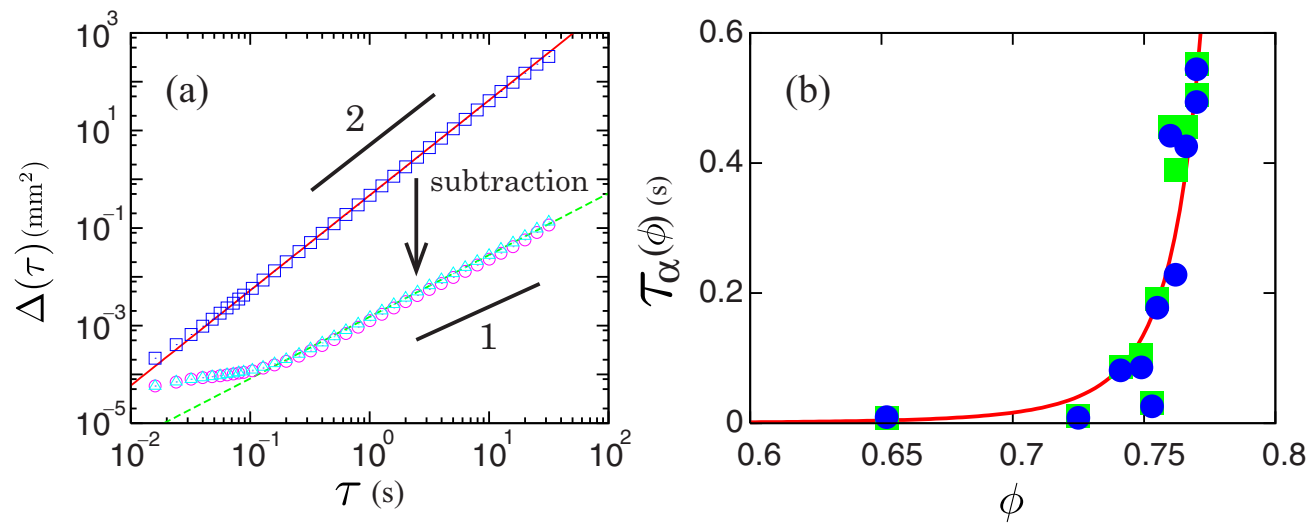

FIG. 2. (Color online) (a) Mean-square displacement $\Delta(\tau)$ of the floaters for $\phi=0.75$. The open squares are obtained without subtracting the displacement $\mathbf{l}_{i}(t)$ due to the large scale convection. The open circles and open triangles are the results with the subtraction, where we use a Gaussian with $d=\sigma$ and a step function with $d=1.6 \sigma$ as coarse graining functions, respectively. Here $\sigma$ is the floater diameter. The red solid and the green dotted lines have slopes 1.9 and 1.1, respectively. (b) Crossover time $\tau_{\alpha}$ plotted against $\phi$, where the closed circles and squares are obtained using the Gaussian $(d=\sigma)$ and the step $(d=1.6 \sigma)$ coarse graining functions, respectively. The solid line represents $\tau_{\alpha} \sim\left(\phi_{c}-\phi\right)^{-3.9}$ with $\phi_{c}=0.82$.

is observed with a typical length scale $\sim 60$ times larger than the floater diameter, which is $\sim 1 / 2$ of the system size, and a typical time scale $\sim 250$ times longer than that of the capillary Faraday waves.

\section{DYNAMIC HETEROGENEITY AND DYNAMIC CRITICALITY}

To focus on microscopic fluctuations, we subtract the displacement due to the large scale convective motion from our experimental data. At first we define the velocity field by the coarse graining method [50-52] as

$$
\mathbf{u}(\mathbf{x}, t)=\frac{\sum_{i} \mathbf{v}_{i}(t) \psi_{d}\left[\left|\mathbf{x}-\mathbf{x}_{i}(t)\right|\right]}{\sum_{i} \psi_{d}\left[\left|\mathbf{x}-\mathbf{x}_{i}(t)\right|\right]},
$$

with the position $\mathbf{x}_{i}(t)$ and the velocity $\mathbf{v}_{i}(t)$ of the $i$ th floater, where we adopt both Gaussian $\left[e^{-(x / d)^{2}}\right]$ and Heaviside $\left[\Theta_{d}(x)=1(x \leqslant d)\right.$ and zero otherwise] as coarse graining kernel functions $\psi_{d}(x)$. Here $d$ is a length scale of the order of the particle diameter. Subsequently we subtract the displacement $\mathbf{l}_{i}(t)=\int_{0}^{t} d s \mathbf{u}\left[\mathbf{x}_{i}(s), s\right]$ due to this macroscopic flow from the position as $\mathbf{r}_{i}(t) \equiv \mathbf{x}_{i}(t)-\mathbf{l}_{i}(t)$ and define an actual displacement during the time interval $\tau$ as $D_{i}(t, \tau) \equiv$ $\left|\mathbf{r}_{i}(t+\tau)-\mathbf{r}_{i}(t)\right|$.

First, we look at single particle dynamics. Approaching high packing fractions, the particles experience caging [33], i.e., a cage composed of a group of particles locally trapped by their neighbors. The particles in the cages are immobile. However, in the presence of fluctuations near the critical packing density, escape jumps occurring at a certain relaxation time create heterogeneous flow, i.e., fast and slow flow regions appear simultaneously [3].

The caging and the successive jumps leave their tracks in the mean-square displacement of individual particles: It has been first theoretically suggested as a measure of the relaxation time for densely packed suspensions exhibiting jamming [39] and then also experimentally confirmed in both a jammed driven granular system [28,39] and colloidal glasses [53]. Unlike glassy systems, in jammed systems there are no jumps and the particles only do jittering motion in the cages $[28,39]$. Therefore, one can observe a very long plateau in the temporal evolution of the mean-square displacement near the jamming packing fraction [28] (or the plateau persists as long as the simulation runs [39]). On the other hand, the plateau in glasses either does not persist very long [5,53] or may not even exist at all near the critical packing fraction (or the glass transition temperature). Moreover, two diffusive regimes are observed [5,53]. For our system, we find very similar behavior to a glass transition: A subdiffusion for short times and an ordinary diffusion at later times. However, instead of observing the finite plateau, we observe an intermediate (a transient) regime to separate the two diffusions.

Figure 2(a) shows the mean-square displacement of the floaters $\Delta(\tau)=\left\langle\sum_{i} D_{i}^{2}(t, \tau) / N\right\rangle_{t}$, where the brackets $\langle\cdots\rangle_{t}$ represent an average over time $t$ and $N$ is the number of floaters in the sample [54]. In our experiment, the floaters are transported by the large scale convection, and thus, the resultant motion is always ballistic. Consequently, when we do not subtract the displacement $\mathbf{l}_{i}(t)$ from the experimental data, $\Delta(\tau)$ quadratically increases with time with a slope 2 in the log-log plot [open squares in Fig. 2(a)]. However, when we do subtract the additional displacement due to the convection for a suitable value of $d$, both the initial subdiffusive and the later diffusive regimes are found [55].

As shown in Fig. 2(b), the crossover time $\tau_{\alpha}$ between these subdiffusive and diffusive regimes, rapidly increases with $\phi$. Since the subdiffusion represents the cage effect of the floaters described above, it is plausible that the crossover time diverges when the system is jammed, where no floater can ever escape from the cage $[28,39]$. On physical grounds, the jamming happens at a critical density $\phi_{c}$, e.g., if the system does not have temperature, $\phi_{c}$ is nearly equal to the random close packing of polydisperse disks, $\phi_{\mathrm{RCP}} \approx 0.84$, while the temperature or external driving force slightly increases $\phi_{c}$ [40]. However, the capillary action keeps floaters at a distance, which might compete against the increase of $\phi_{c}$ due to the 

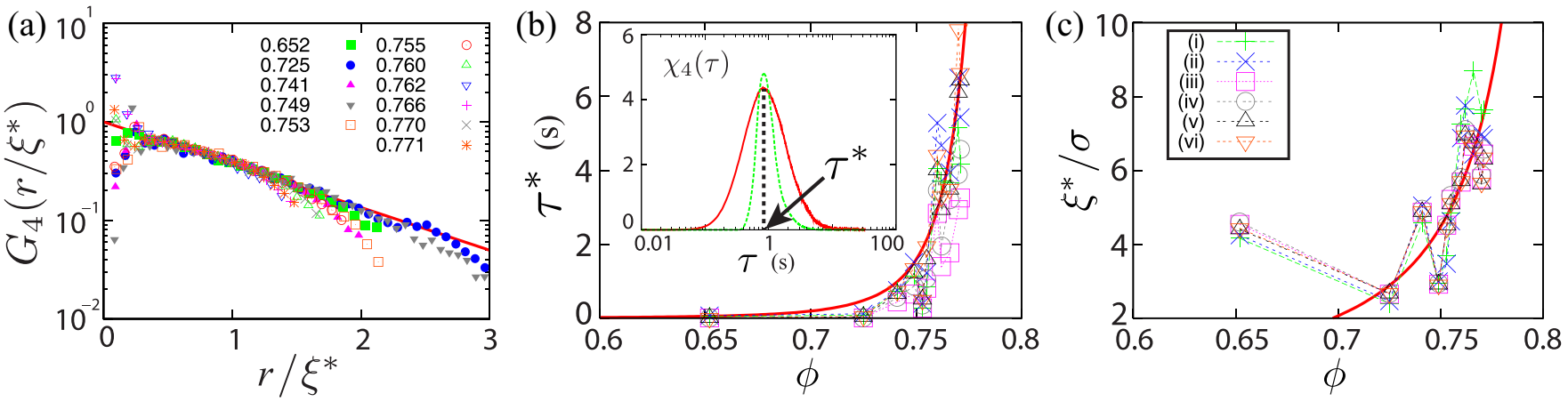

FIG. 3. (Color online) (a) The scaled four-point correlation function $G_{4}\left(r / \xi^{*}\right) \equiv\left(r / \xi^{*}\right)^{\beta} g_{4}\left(r, \tau^{*}\right) / A$ where we use Gaussians for the coarse graining and overlap functions respectively [case (iii) in Table I] and the solid line represents $e^{-r / \xi^{*}}$. (b) The time scale of the dynamic heterogeneity $\tau^{*}$ vs $\phi$ obtained from the four-point dynamic susceptibility $\chi_{4}(\tau)$. The solid line represents $\tau^{*} \sim\left(\phi_{c}-\phi\right)^{-3.9}$. Inset: $\chi_{4}(\tau)$ for $\phi=0.75$, where the green dotted line is obtained without subtracting the convective displacement $\mathbf{l}_{i}(t)$ [case (i) in Table I] and the red solid line with the subtraction [case (iii) in Table I]. (c) The dynamic correlation length $\xi^{*}$ obtained from $g_{4}(r, \tau)$ for $\tau=\tau^{*}$. The solid line represents $\xi^{*} \sim\left(\phi_{c}-\phi\right)^{-1.4}$. The legend in (c) indicates the conditions described in Table I and explains the symbols in (b) and (c).

external driving [56]. In addition, $\phi_{c}$ needs to be considerably larger than $\phi_{\text {expt }}=0.77$, the largest experimental average at which we are able to measure, so that we can assume the critical density is in the range $\phi_{\text {expt }}=0.77<\phi_{c}<\phi_{\mathrm{RCP}}$.

By fitting a power law $\tau_{\alpha} \sim\left(\phi_{c}-\phi\right)^{\alpha}$ to our data [57] we find that $\phi_{c} \approx 0.82$, which is consistent with the above and leads us to conclude that $\phi_{c}=0.82 \pm 0.02$. It is worth mentioning that previous experiments found a similar divergence of the relaxation time (associated with the contact number) near the critical density $0.8151[28,33]$. Note that this value is considerably larger than the suggested static buckling density of the attractive monodisperse spheres [58], namely $\phi_{b} \simeq 0.71$. Next, we use this fixed value for $\phi_{c}$ in our power-law fit to obtain the exponent $\alpha \simeq-3.9 \pm 0.9$, which is consistent with the exponent $\alpha \simeq-4.0 \pm 0.6$ found in an earlier experiment [27].

To quantify the heterogenous dynamics of the floaters, we introduce the self-overlap order parameter $q_{a}(t, \tau)=$ $\sum_{i} w_{a}\left[D_{i}(t, \tau)\right] / N$ and the four-point dynamic susceptibility $\chi_{4}(\tau)=N\left[\left\langle q_{a}^{2}(t, \tau)\right\rangle_{t}-\left\langle q_{a}(t, \tau)\right\rangle_{t}^{2}\right]$. Here $w_{a}(x)$ is the overlap function defined as a Gaussian $e^{-(x / a)^{2}}$ or a Heaviside step function $\Theta_{a}(x)$ as defined previously ( 1 for $x \leqslant a$ and 0 , otherwise). The width of the overlap function $a$ is a measure for the typical distance over which a single floater can move within time $\tau$. To disregard the motion of the floaters in the cages, $a$ is chosen to be larger than their typical displacement inside a cage and also chosen to maximize [19] the extremal value of the $\chi_{4}(\tau)$ as shown in the inset of Fig. 3(b).

The various coarse graining functions and overlap functions in total give us six different manners of analyzing the data, if we also include the possibility of not subtracting the displacement due to the large scale convection before the dynamic heterogeneity analysis. These are summarized in Table I, together with the optimal values of $d$ and $a$ obtained as described above [59]. When we plot the $\chi_{4}(\tau)$ we obtain similar results in all six cases [case (i) in Table I is shown in the inset of Fig. 3(b)]. In particular the location of the peak in $\chi_{4}(\tau)$ provides us with an estimate of the typical time scale $\tau^{*}$ of the dynamic heterogeneity, which are plotted for all six cases as functions of $\phi$ in Fig. 3(b).
To investigate the dynamic correlation length of the floaters, we apply the four-point correlation function [60]

$$
g_{4}(r, \tau)=\frac{1}{2 \pi r N}\left\langle\sum_{i, j} \delta\left[r-r_{i j}(t)\right] c_{i j}(t, \tau)\right\rangle_{t}-\rho\left\langle q_{a}(t, \tau)\right\rangle_{t}^{2}
$$

satisfying $\chi_{4}(\tau)=2 \pi \int r g_{4}(r, \tau) d r$, where $\rho \equiv N / S$ and $S$ are the number density of the floaters and the area of interest, respectively. $N$ is the number of floaters as introduced previously. In addition, we define $r_{i j}(t) \equiv\left|\mathbf{r}_{i}(t)-\mathbf{r}_{j}(t)\right|$ and $c_{i j}(t, \tau) \equiv w_{a}\left[D_{i}(t, \tau)\right] w_{a}\left[D_{j}(t, \tau)\right]$. Furthermore, we assume the Ornstein-Zernike form of the four-point correlation function [60], in which the dynamic correlation length $\xi^{*}$ is obtained considering the scaling $g_{4}\left(r, \tau^{*}\right)=A\left(r / \xi^{*}\right)^{-\beta} e^{-r / \xi^{*}}$ for some amplitude $A$ and exponent $\beta$, where $\tau^{*}$ is the time scale obtained from $\chi_{4}(\tau)$.

Figure 3(a) shows the function $G_{4}\left(r / \xi^{*}\right) \equiv$ $\left(r / \xi^{*}\right)^{\beta} g_{4}\left(r, \tau^{*}\right) / A$, where the (very weak) exponent $\beta=0.01$ is taken to be independent of $\phi$. The resultant $G_{4}\left(r / \xi^{*}\right)$ successfully collapses onto a single master curve $e^{-r / \xi^{*}}$ for each $\phi$ except for the tails. This procedure is repeated for each condition in Table I. Remarkably, we find that neither the value of the exponent nor the master curve presents any significant difference.

Figure 3 displays the time scale of the dynamic heterogeneity $\tau^{*}$ [Fig. 3(b)] and the dynamic correlation length

TABLE I. Analysis methods: The set of the coarse graining functions and the overlap functions, with $d$ and $a$ as described in the main text, both in terms of the floater diameter $\sigma$.

\begin{tabular}{lcccc}
\hline \hline & Coarse graining function & Overlap function & $d / \sigma$ & $a / \sigma$ \\
\hline (i) & none & Gaussian & - & 0.49 \\
(ii) & none & Heaviside & - & 0.52 \\
(iii) & Gaussian & Gaussian & 1.0 & 0.038 \\
(iv) & Gaussian & Heaviside & 1.0 & 0.042 \\
(v) & Heaviside & Gaussian & 1.6 & 0.042 \\
(vi) & Heaviside & Heaviside & 1.6 & 0.046 \\
\hline \hline
\end{tabular}


$\xi^{*}$ [Fig. 3(c)], where both are plotted versus $\phi$ and increase strongly with $\phi$. One can introduce the power-law fits [1719,27,37-39]

$$
\begin{aligned}
\tau^{*} & =C\left(\phi_{c}-\phi\right)^{\eta}, \\
\xi^{*} & =D\left(\phi_{c}-\phi\right)^{\lambda} .
\end{aligned}
$$

Both the time exponent $\eta$ and the length exponent $\lambda$ are calculated considering all conditions reported in Table I. Fitting to the data we obtain $\eta \simeq-3.9 \pm 0.4$ and $\lambda \simeq-1.4 \pm$ 0.4 for each condition in Table I where we again used $\phi_{c} \simeq$ $0.82 \pm 0.02$. Finally, combining Eqs. (3) and (4) in the light of dynamic criticality [17-19,37-39], namely $\tau^{*} \sim \xi^{* \nu}$, we quantify the relation between $\eta$ and $\lambda$ as $v=\eta / \lambda \simeq 2.7 \pm 1.2$.

\section{CONCLUSION}

Studying agitated floaters by the capillary Faraday waves, after eliminating their naturally occurring large-scale convection, their mean-square displacement dynamics resembles caging as observed in glassy liquids: An initial subdiffusion in earlier (short) times is followed by an ordinary diffusion for later times. The crossover time $\tau_{\alpha}(\phi)$ between the two regimes [Fig. 2(b)] drastically increases near the estimated critical density $\phi_{c}$.

Although we observe that the increase can be explained by many functional forms, we force to fit a power law to make the connection to the literature of glasses and jammed systems, where power laws are both expected and traditionally used for this purpose. Therefore, even though the fit of $\tau_{\alpha}(\phi)$ works well, we can only claim an apparent consistency. Thus, $\tau_{\alpha}(\phi)$ is fitted by a power law $\left(\phi_{c}-\phi\right)^{\alpha}$ with exponent $\alpha \simeq-3.9$. In addition, a second time scale $\tau^{*}$ is that of the four-point dynamic susceptibility $\chi_{4}(\tau)$ [Fig. 3(b)], which is also fitted by a power law which remarkably has the very same exponent $\eta \simeq-3.9$.

The typical distance between two correlated, successive events, the dynamic correlation length $\xi^{*}$ [Fig. 3(c)] obtained from the scaled four-point correlation function $G_{4}\left(r / \xi^{*}\right)$ [Fig. 3(a)], presents a power-law scaling with exponent $\lambda \simeq$ -1.4 . Both of our dynamic exponents, $\eta$ and $\lambda$, are in a good agreement with the previous experiment on sheared microgel spheres by Nordstrom et al. [27], where the critical exponents of the time and length scales were found to be -4 and $-4 / 3$, respectively.

The coarse graining procedure allows us to successfully remove the convective mean flow, i.e., the large scale collective motion observed for each $\phi$. While this is indeed necessary to study microfluctuations driven by diffusion, paradoxically, one of our main results is that it is not necessary to remove this mean flow for $\chi_{4}(\tau)$ and $g_{4}(r, \tau)$. These results only depend insignificantly on whether the mean flow is subtracted or not, which has not been indicated in previous reported analysis [28]. In addition, the results do hardly depend on the choice of the coarse graining and overlap functions, as long as the length scales in these test functions are optimized under physical constraints, e.g., the particle diameter, the typical particle displacement inside a cage, etc. In fact, from Table I it can be appreciated that the coarse-graining (smoothing) length $a$ must be an order of magnitude lower with the convective mean flow subtraction $(a \simeq 0.04 \sigma)$ than without $(a \simeq 0.5 \sigma)$.

Finally, we determine from the fits that $\phi_{c}=0.82$, while close to the two-dimensional random close packing $\left(\phi_{\mathrm{RCP}} \simeq\right.$ 0.84 ), being considerably larger than the suggested critical density for static monodisperse floaters $\left(\phi_{b}=0.71\right)$, and also larger than the largest packing density that we could reach experimentally, namely $\phi_{\text {expt }} \simeq 0.77$. For larger $\phi$, our layer of floating spheres is not stable under driving. Understanding the difference between $\phi_{\text {expt }} \simeq 0.77$ and $\phi_{c}$ requires further study. In this respect, investigating the origins of the dynamic heterogeneity, of which it is known that there are many [33], can give further information about our system and help to clarify this difference.

\section{ACKNOWLEDGMENTS}

We thank O. Dauchot, D. J. Durian, T. Hatano, H. Hayakawa, H. Katsuragi, T. Kawasaki, D. Lohse, K. Miyazaki, Y. Tagawa, and R. Yamamoto for fruitful discussions. This work is partially supported by the NWO-STW VICI Grant 10828 and is part of the research program of the FOM, which is financially supported by NWO; K. Saitoh and C. Sanlı acknowledge financial support. C. Sanl acknowledges supports from the Okinawa Inst. of Sci. and Tech. Graduate University (OIST), the Facultés Universitaires Notre-Dame de la Paix (FUNDP, the University of Namur), and the European Union 7th Framework Programme OptimizR Project.
[1] R. K. Pathria, Statistical Mechanics (Butterworth-Heinemann, Oxford, 1996).

[2] L. Berthier and J. Kurchan, Nat. Phys. 9, 310 (2013).

[3] M. D. Ediger, Annu. Rev. Phys. Chem. 51, 99 (2000).

[4] L. Berthier, G. Biroli, J.-P. Bouchaud, W. Kob, K. Miyazaki, and D. R. Reichman, J. Chem. Phys. 126, 184503 (2007).

[5] L. Berthier and G. Biroli, Rev. Mod. Phys. 83, 587 (2011).

[6] G. Biroli and J. P. Garrahan, J. Chem. Phys. 138, 12A301 (2013).

[7] L. Berthier, Physics 4, 42 (2011).

[8] H. Mizuno and R. Yamamoto, J. Chem. Phys. 136, 084505 (2012).

[9] A. J. Liu and S. R. Nagel, Nature (London) 396, 21 (1998).
[10] C. S. O’Hern, S. A. Langer, A. J. Liu, and S. R. Nagel, Phys. Rev. Lett. 88, 075507 (2002).

[11] C. S. O’Hern, L. E. Silbert, A. J. Liu, and S. R. Nagel, Phys. Rev. E 68, 011306 (2003).

[12] T. S. Majmudar, M. Sperl, S. Luding, and R. P. Behringer, Phys. Rev. Lett. 98, 058001 (2007).

[13] M. van Hecke, J. Phys.: Condens. Matter 22, 033101 (2010).

[14] T. Narumi, S. V. Franklin, K. W. Desmond, M. Tokuyama, and E. R. Weeks, Soft Matter 7, 1472 (2011).

[15] Y. Rahmani, K. van der Vaart, B. van Dam, Z. Hu, V. Chikkadi, and P. Schall, Soft Matter 8, 4264 (2012). 
[16] O. Dauchot, G. Marty, and G. Biroli, Phys. Rev. Lett. 95, 265701 (2005).

[17] A. S. Keys, A. R. Abate, S. C. Glotzer, and D. J. Durian, Nat. Phys. 3, 260 (2007).

[18] A. R. Abate and D. J. Durian, Phys. Rev. E 76, 021306 (2007).

[19] F. Lechenault, O. Dauchot, G. Biroli, and J. P. Bouchaud, Europhys. Lett. 83, 46003 (2008).

[20] F. Lechenault, R. Candelier, O. Dauchot, J. P. Bouchaud, and G. Biroli, Soft Matter 6, 3059 (2010).

[21] P. Mayer, H. Bissig, L. Berthier, L. Cipelletti, J. P. Garrahan, P. Sollich, and V. Trappe, Phys. Rev. Lett. 93, 115701 (2004).

[22] B. J. Gluckman, C. B. Arnold, and J. P. Gollub, Phys. Rev. E 51, 1128 (1995).

[23] D. Vella and L. Mahadevan, Am. J. Phys. 73, 817 (2005).

[24] D. Y. C. Chan, J. J. D. Henry, and L. R. White, J. Colloid Interface Sci. 79, 410 (1981).

[25] C. Sanl1, D. Lohse, and D. van der Meer, Phys. Rev. E 89, 053011 (2014).

[26] O. Herbst, R. Cafiero, A. Zippelius, H. J. Herrmann, and S. Luding, Phys. Fluids 17, 107102 (2005).

[27] K. N. Nordstrom, J. P. Gollub, and D. J. Durian, Phys. Rev. E 84, 021403 (2011).

[28] C. Coulais, R. P. Behringer, and O. Dauchot, Soft Matter 10, 1519 (2014).

[29] L. Berthier, Phys. Rev. E 69, 020201(R) (2004).

[30] L. Berthier, G. Biroli, J.-P. Bouchaud, L. Cipelletti, D. E. Masri, D. L'Hôte, F. Ladieu, and M. Pierno, Science 310, 1797 (2005).

[31] L. Berthier, G. Biroli, J. P. Bouchaud, L. Cipelletti, and W. van Saarloos, Dynamical Heterogeneities in Glasses, Colloids, and Granular Media (Oxford University Press, Oxford, 2011).

[32] T. Hatano, J. Phys.: Conf. Ser. 319, 012011 (2011).

[33] C. Coulais, R. Candelier, and O. Dauchot, AIP Conf. Proc. 1542, 25 (2013).

[34] G. Marty and O. Dauchot, Phys. Rev. Lett. 94, 015701 (2005).

[35] H. Katsuragi, A. R. Abate, and D. J. Durian, Soft Matter 6, 3023 (2010).

[36] A. R. Abate and D. J. Durian, Phys. Rev. E 74, 031308 (2006).

[37] P. M. Chaikin and T. C. Lubensky, Principles of Condensed Matter Physics (Cambridge University Press, Cambridge, 1995).

[38] H. Nishimori and G. Ortiz, Elements of Phase Transitions and Critical Phenomena (Oxford University Press, Oxford, 2011).

[39] A. Ikeda, L. Berthier, and G. Biroli, J. Chem. Phys. 138, 12A507 (2013).

[40] L. Berthier and T. A. Witten, Phys. Rev. E 80, 021502 (2009).

[41] T. Hatano, Phys. Rev. E 79, 050301(R) (2009).

[42] B. P. Tighe, E. Woldhuis, J. J. C. Remmers, W. van Saarloos, and M. van Hecke, Phys. Rev. Lett. 105, 088303 (2010).

[43] T. Hatano, Prog. Theor. Phys. Suppl. 184, 143 (2010).

[44] S. Douady, J. Fluid Mech. 221, 383 (1990).
[45] The particles have been custom made by a collaborating company and are not commercially available.

[46] S. Luding, Adv. Complex Syst. 04, 379 (2001).

[47] C. Duez, C. Ybert, C. Clanet, and L. Bocquet, Nat. Phys. 3, 180 (2007).

[48] Even in the dilute case, the distance $r$ between the floaters is much smaller than the capillary length $L_{c}=\sqrt{\gamma / \rho_{l} g}$, with $\gamma$ as the surface tension coefficient of the interface, $\rho_{l}$ as the liquid density, and $g$ as the acceleration of gravity. For an air-water interface at $20^{\circ} \mathrm{C}, L_{c}=2.7 \mathrm{~mm}$.

[49] P. Cicuta and D. Vella, Phys. Rev. Lett. 102, 138302 (2009).

[50] I. Goldhirsch, Granular Matter 12, 239 (2010).

[51] T. Weinhart, A. R. Thornton, S. Luding, and O. Bokhove, Granular Matter 14, 289 (2012).

[52] C. Goldenberg and I. Goldhirsch, Handbook of Theoretical and Computational Nanotechnology (American Scientific, Stevenson Ranch, CA, 2006).

[53] A. Ghosh, V. K. Chikkadi, P. Schall, J. Kurchan, and D. Bonn, Phys. Rev. Lett. 104, 248305 (2010).

[54] The procedure in the ensemble average is to calculate $\mid \mathbf{r}_{i}(t+$ $\tau)-\mathbf{r}_{i}(t) \mid$ using arbitrary starting times $t$ and averaging over $t$. A similar procedure will be followed in calculating ensemble averages in the self-overlap order parameter, four-point dynamic susceptibility, and four-point correlation function.

[55] The optimal values for $d$ were obtained as follows: When looking at the subtraction procedure as a function of $d$ we find that the displacement rises steeply from zero for $d \ll \sigma, \sigma$ is the floater diameter, into a plateau from which it continues to rise. A value in the center of the plateau is chosen, which happens to correspond roughly to the floater diameter.

[56] From the (two-dimensional) pair correlation function $g(r)$ we observe no evidence for significant crystallization which may cause an increase of this upper limit for $\phi_{c}$. Second, although buckling may be a significant factor, it does not lead to a broadening of the first peak in $g(r)$ that one would expect to be present if particles start to overlap for increasing $\phi$. And, finally, the homogenized local packing fraction shows a sharp cutoff at $\phi \approx 0.84$. These facts together suggests that $\phi_{c}<0.84$.

[57] Due to their limited range other functional forms could possibly also fit our dynamic time and length scales. However, we restrict ourselves to power-law fits to compare our results to those in the literature.

[58] M. Berhanu and A. Kudrolli, Phys. Rev. Lett. 105, 098002 (2010).

[59] Both $d$ and $a$ are first calculated for each $\phi$ separately, and then appropriate values are obtained by averaging over the determined values.

[60] N. Lačević, F. W. Starr, T. B. Schrøder, and S. C. Glotzer, J. Chem. Phys. 119, 7372 (2003). 